\title{
Quasi-classical Lie algebras and their contractions
}

\author{
R. Campoamor-Stursberg $\dagger$ \\ † Dpto. Geometría y Topología \\ Fac. CC. Matemáticas \\ Universidad Complutense de Madrid \\ Plaza de Ciencias, 3 \\ E-28040 Madrid, Spain \\ E-mail: rutwig@mat.ucm.es
}

\begin{abstract}
After classifying indecomposable quasi-classical Lie algebras in low dimension, and showing the existence of non-reductive stable quasi-classical Lie algebras, we focus on the problem of obtaining sufficient conditions for a quasi-classical Lie algebras to be the contraction of another quasi-classical algebra. It is illustrated how this allows to recover the Yang-Mills equations of a contraction by a limiting process, and how the contractions of an algebra may generate a parameterized families of Lagrangians for pairwise non-isomorphic Lie algebras.
\end{abstract}

PACS numbers: 02.20Sv, 02.20Qs, 11.15Kc 


\section{Introduction}

In many physical applications one is often confronted to consider Lie algebras other than semisimple and endowed with a non-degenerate symmetric bilinear form that is associative with respect to the bracket (Bohr and Bucher, 1986; Das, 1989; Schimming and Mundt, 1992). For the classical case, the Killing metric tensor provides this form, with the additional advantage of being related to the adjoint representation (Popov, 1991). However, even for reductive Lie algebras this approach fails, and we have to consider a different representation, due to degeneracy of the trace form. This suggests to consider the problem in general, which gives rise to the class of quasi-classical Lie algebras (Okubo, 1979). This approach allows for example to treat non-abelian YangMills gauge theories in unified manner, covering the abelian and semisimple cases, and even extending it to the solvable case (Slavnov and Faddeev, 1978; Okubo and Kamiya, 2002). The existence of a bilinear form with the required properties can be characterized, like in the semisimple case, by the existence of a quadratic Casimir operator of a certain form (Casimir, 1931; Okubo, 1979). Although it has been proved in that only those gauge theories based upon compact algebras remain ghost-free when quantized, the general case still remains of interest for the analysis of solutions of the Yang-Mills equations (Das 1989; Mundt, 1993).

In the generalized theory, the classical Lagrangian is replaced by

$$
L(x)=g^{i j} F_{\mu \nu, i} F_{, j}^{\mu \nu},
$$

where $g^{i j}$ are the components of the non-degenerate form on $\mathfrak{g}$. Given an element $Y \in \mathfrak{g}$, it is straightforward to verify that for any $X, Y \in \mathfrak{g}$ we have the invariance condition

$$
\left((\exp Y)^{-1} X_{i}(\exp Y),(\exp Y)^{-1} X_{j}(\exp Y)\right)=\left(X_{i}, X_{j}\right),
$$

thus, taking a function $\eta^{i}(x)$ of the spacetime coordinate $x$ and defining $g(x)=$ $\exp \left(\eta^{i}(x) X_{i}\right)$, the local transformations defined by

$$
\begin{aligned}
& A_{\mu}^{\prime}=g^{-1}(x) A_{\mu}(x) g(x)-g^{-1}(x) \partial_{\mu} g(x), \\
& F_{\mu \nu}^{\prime}=g^{-1}(x) F_{\mu \nu}(x) g(x)
\end{aligned}
$$

leave the Lagrangian (11) invariant and consistently reproduce the equations of motion

$$
\left[A_{\mu}(x), A_{\nu}(x)\right]+\partial^{\lambda} F_{\lambda \mu}(x)=0
$$

where, as usual,

$$
\begin{aligned}
& A_{\mu}(x)=X_{a} A_{\mu}^{a}(x), \\
& F_{\mu \nu}(x)=\partial_{\mu} A_{\nu}(x)-\partial_{\nu} A_{\mu}(x)+\left[A_{\mu}(x), A_{\nu}(x)\right] .
\end{aligned}
$$

Solutions of these equations for nilpotent and solvable Lie algebras have been analyzed for various types of Lie algebras in different situations, like the curvature zero case $F_{\mu \nu}=0$ or the sourceless case with constant potentials (Bollini and Giambigi, 1984; Schimming and Mundt 1992). 
In this work we focus on the properties of quasi-classical Lie algebras with respect to contractions. We point out that non-abelian quasi-classical algebras can arise as contractions of Lie algebras $\mathfrak{g} \rightsquigarrow \mathfrak{g}^{\prime}$ that do not carry a non-degenerate form, or even don't possess Casimir operators. However, if the quadratic Casimir operator is the result of a limiting process of a quadratic invariant of the contracted algebra, then both algebras are quasi-classical. This will allow us to deduce the gauge fields and the Lagrangian of the contraction by limits of the corresponding quantities over $\mathfrak{g}$.

We first analyze some structural properties of Lie algebras of this type, especially quasi-classical Lie algebras that are either nilpotent or have a nontrivial Levi decomposition. The analysis in low dimension suggests that contractions of reductive (especially semisimple) Lie algebras provide all quasi-classical non-abelian Lie algebras. This is however not true in general, as will be established by a counterexample of a stable quasi-classical Lie algebras in dimension 10. In section 4 we study under which conditions a non-abelian quasi-classical Lie algebra arises as the contraction of another Lie algebra with the same property. These results are applied to the contraction procedure of the Yang-Mills equations for contractions that preserve the property of being quasi-classical.

\section{Quasi classical Lie algebras}

Let $\mathfrak{g}$ be a Lie algebra and (.,.) a symmetric bilinear form that satisfies the associativity condition

$$
(X,[Y, Z])=([X, Y], Z), \quad \forall X, Y, Z \in \mathfrak{g} .
$$

The form is non-degenerate if the radical $\mathfrak{R}=\{X \in \mathfrak{g} \mid(X, Y)=0, \forall Y\}$ reduces to zero. Following the notations of (Okubo, 1979), we call a Lie algebra $\mathfrak{g}$ quasi-classical (short QCLA) if it possesses a bilinear symmetric non-degenerate form (., .) that satisfies equation (77). It follows at once that any reductive Lie algebra, i.e., any direct sum of a semisimple and abelian Lie algebra, is quasi-classical. In (Okubo, 1998) it was shown that the most general non-abelian gauge theory is based on QCLAs.

In particular, a characterization of QCLAs in terms of quadratic operators can be given (Okubo, 1979), in complete analogy to the classical semisimple case proved in (Casimir, 1931):

Proposition 1 A Lie algebra $\mathfrak{g}$ is quasi-classical if and only if it possesses a quadratic Casimir operator $C_{2}=g^{a b} X_{a} X_{b}$ such that the symmetric matrix $g^{a b}$ satisfies the constraint

$$
g^{a b} g_{b c}=\delta_{a c},
$$

where $g_{a b}$ is the inverse of $g^{a b}$.

Those properties of QCLA not using explicitly the adjoint representation of the algebra constitute natural generalizations of those observed for the Killing tensor in 
semisimple Lie algebras. Among the elementary properties of quasi-classical algebras, we enumerate the following three, the proof of which is completely analogous to the semisimple case with the Killing metric tensor:

(i) If $\mathfrak{g}$ is quasi-classical complex, then any of its real forms is quasi-classical.

(ii) If $\mathfrak{g}_{1}$ and $\mathfrak{g}_{2}$ are quasi-classical, then their direct sum is also quasi-classical.

(iii) If a quasi-classical Lie algebra $\mathfrak{g}$ admits an ideal $\mathfrak{I}$ such that $\left.(.,)\right|_{.\mathfrak{I}}$ is nondegenerate, then $\mathfrak{I}^{\perp}=\{X \in \mathfrak{g} \quad \mid \quad(X, \mathfrak{I})=0\}$ is also a quasi-classical ideal and $\mathfrak{g}$ is decomposable.

Lemma 1 If the quasi-classical Lie algebra $\mathfrak{g}=\mathfrak{g}_{1} \oplus \mathfrak{g}_{2}$ is decomposable and the restriction of $(.,$.$) to the centre Z(\mathfrak{g})$ is degenerate, then both $\mathfrak{g}_{1}$ and $\mathfrak{g}_{2}$ are quasi-classical algebras.

Proof. If $\mathfrak{g}_{1}$ were not quasi-classical, then there exists a nonzero element $z \notin\left[\mathfrak{g}_{1}, \mathfrak{g}_{1}\right]$ such that $(x, z)=0 \forall x \in \mathfrak{g}_{1}$. In particular, $z$ is not in the centre of $\mathfrak{g}$. Let $y \in \mathfrak{g}_{1}$ such that $[x, y] \neq 0$. By non-degeneracy, there exists an $x \in \mathfrak{g}$ such that $(x,[y, z])=([x, y]) \neq$,0 . By the decomposition, $x$ belongs to $\mathfrak{g}_{1}$ and therefore $[x, y] \in\left[\mathfrak{g}_{1}, \mathfrak{g}_{1}\right]$, contradicting the choice of $z$.

These properties reduce the classification of quasi-classical Lie algebras to the analysis of indecomposable Lie algebras, i.e., those which do not decompose as a direct sum of ideals. We also remark that property (iii) above does not exclude the possibility that a QCLA has quasi-classical ideals, but refers to the induced bilinear form on the ideal.

Proposition 2 Let $R$ be a representation of a semisimple Lie algebra $\mathfrak{s}$ such that the multiplicity of the trivial representation $\Gamma_{0}$ in $R$ is zero. If $\mathfrak{g} \vec{\oplus}_{R}(\operatorname{dim} R) L_{1}$ is quasi-classical, then the restriction of the inner product (.,.) to the abelian radical $\mathfrak{r}=(\operatorname{dim} R) L_{1}$ is degenerate.

Proof. Since mult $\Gamma_{0} R=0$, for any $Y$ in the radical there exists $X \in \mathfrak{s}$ and $Y^{\prime}$ in the radical such that $Y=\left[X, Y^{\prime}\right]$. By the associativity of the bilinear form (.,.) we have

$$
\left(\left[X, Y_{i}\right], Y_{j}\right)=\left(\mathfrak{r}, Y_{j}\right)=\left(X,\left[Y_{i}, Y_{j}\right]\right)=0,
$$

showing that the restriction $\left.(.,)\right|_{.\mathfrak{r}}$ to the radical is degenerate.

As a consequence of this result, no non-degenerate inner product in the abelian Lie algebra $(\operatorname{dim} R) L_{1}$ can be extended to the semidirect product $\mathfrak{g} \vec{\oplus}_{R}(\operatorname{dim} R) L_{1}$ without violating the associativity condition (7).

Corollary 1 If mult $_{\Gamma_{0}} R=0$ and $\mathfrak{g} \vec{\oplus}_{R}(\operatorname{dim} R) L_{1}$ is a $Q C L A$, then $\operatorname{dim} R \leq \operatorname{dim} \mathfrak{s}$.

Trivial examples of algebras having nontrivial Levi decomposition and being quasiclassical are the semidirect products $\mathfrak{s} \vec{\oplus}_{a d \mathfrak{s}}(\operatorname{dim} \mathfrak{s}) L_{1}$ (Campoamor-Stursberg, 2003b). We point out that the preceding corollary does not hold if the radical is not abelian. 
Proposition 3 Let $\mathfrak{g}$ be a indecomposable quasi-classical Lie algebra of dimension $n \leq 9$ and having a nontrivial Levi subalgebra. Then $\mathfrak{g}$ is isomorphic to one of the following Lie algebras

(i) $L_{6,1}=\mathfrak{s o}(3) \vec{\oplus}_{a d} 3 L_{1}$ with structure tensor

$$
\begin{aligned}
& C_{12}^{3}=1, \quad C_{13}^{2}=-1, \quad C_{23}^{1}=1, \quad C_{15}^{6}=1, \quad C_{16}^{5}=-1, \quad C_{24}^{6}=-1, \\
& C_{26}^{4}=1, \quad C_{34}^{5}=1, \quad C_{35}^{4}=-1 \text {. }
\end{aligned}
$$

(ii) $L_{6,4}=\mathfrak{s l}(2, \mathbb{R}) \vec{\oplus}_{a d} 3 L_{1}$ with structure tensor

$$
\begin{aligned}
& C_{12}^{2}=2, \quad C_{13}^{3}=-2, \quad C_{23}^{1}=1, \quad C_{14}^{4}=2, \quad C_{16}^{6}=-2, \quad C_{25}^{4}=2, \\
& C_{26}^{5}=1, \quad C_{34}^{5}=1, \quad C_{35}^{6}=2 .
\end{aligned}
$$

(iii) $L_{9,11}=\mathfrak{s o}(3) \vec{\oplus}_{2 a d} \mathcal{A}_{6,3}$ with structure tensor

$$
\begin{aligned}
& C_{12}^{3}=1, \quad C_{13}^{2}=-1, \quad C_{23}^{1}=1, \quad C_{15}^{6}=1, \quad C_{16}^{5}=-1, \quad C_{18}^{9}=1, \\
& C_{19}^{8}-1, \quad C_{24}^{6}=-1, \quad C_{26}^{4}=1, \quad C_{27}^{9}=-1, \quad C_{29}^{7}=1, \quad C_{34}^{5}=1, \\
& C_{35}^{4}=-1, \quad C_{37}^{8}=1, \quad C_{38}^{7}=-1, \quad C_{45}^{9}=1, \quad C_{46}^{8}=-1, \quad C_{56}^{7}=1 .
\end{aligned}
$$

(iv) $L_{9,62}=\mathfrak{s l}(2, \mathbb{R}) \vec{\oplus}_{2 a d} A_{6,3}$ with structure tensor

$$
\begin{aligned}
& C_{12}^{2}=2, \quad C_{13}^{3}=-2, \quad C_{23}^{1}=1, \quad C_{14}^{4}=2, \quad C_{16}^{6}=-2, \quad C_{17}^{7}=2, \\
& C_{19}^{9}=-2, \quad C_{25}^{4}=2, \quad C_{26}^{5}=1, \quad C_{28}^{7}=2, \quad C_{29}^{8}=1, \quad C_{34}^{5}=1, \\
& C_{35}^{6}=2, \quad C_{37}^{8}=1, \quad C_{38}^{9}=2, \quad C_{45}^{7}=2, \quad C_{46}^{8}=1, \quad C_{56}^{9}=2 .
\end{aligned}
$$

where $\left[X_{i}, X_{j}\right]=C_{i j}^{k} X_{k}$ over the basis $\left\{X_{1}, . ., X_{n}\right\}$ of $\mathfrak{g}$.

We remark that both nine dimensional algebra have the same complexification, and have indeed a quasi-classical radical. The proof follows from the classification of Lie algebras with nontrivial Levi decomposition of (Turkowski, 1988; 1992) and the analysis of their invariants (Campoamor-Stursberg, 2003a).

For solvable quasi-classical Lie algebras various general constructions exists (see e.g. (Okubo and Kamiya, 2002; Myung, 1986), while the nilpotent case was analyzed in (Favre and Santharoubane, 1987). We now prove that a non-degenerate quadratic Casimir operator imposes some restrictions on the nilindex of a nilpotent Lie algebra.

Proposition 4 A nilpotent quasi-classical Lie algebra $\mathfrak{n}$ of dimension $n$ has at most nilindex $n-2$.

Proof. If $\mathfrak{n}$ is nilpotent of nilindex $n-1$, then we can always find a basis $\left\{X_{1}, \ldots, X_{n}\right\}$ such that $\left[X_{1}, X_{i}\right]=X_{i+1}$ for $2 \leq i \leq n-1$. Realizing the Lie algebra by differential operators $\widehat{X}_{i}=C_{i j}^{k} x_{k} \frac{\partial}{\partial x_{j}}$ in $C^{\infty}\left(\mathfrak{n}^{*}\right)$, the obtainment of Casimir operators is equivalent to obtain the polynomial solutions $F\left(x_{1}, . ., x_{n}\right)$ of the system of PDEs $\widehat{X}_{i} F=0$ and symmetrize them (Trofimov, 1983). If we consider the differential operator associated to $X_{1}$ :

$$
\widehat{X}_{1}(F)=\sum_{k=2}^{n-1} x_{k+1} \frac{\partial F}{\partial x_{k}}=0
$$


the equation (9) has the general quadratic solution

$C_{2}(2 m)=a_{0} x_{1}^{2}+\sum_{k=1}^{m-1} a_{k}\left(\frac{1}{2} x_{m+k}^{2}+\sum_{j=1}^{m-k}(-1)^{j} x_{m+k-j} x_{m+k+j}\right)+a_{m} x_{1} x_{2 m}+a_{m+1} x_{2 m}^{2}$

if $n=2 m$, and

$C_{2}(2 m-1)=a_{0} x_{1}^{2}+\sum_{k=1}^{m-2} a_{k}\left(\frac{1}{2} x_{m+k}^{2}+\sum_{j=1}^{m-k-1}(-1)^{j} x_{m+k-j} x_{m+k+j}\right)+a_{m} x_{1} x_{2 m-1}+a_{m+1} x_{2 m-1}^{2}$,

if $n=2 m-1$. For the latter solution we see that $\frac{\partial C_{2}(2 m-1)}{\partial x_{2}}=0$, thus we never obtain a non-degenerate quadratic Casimir operator. It remains to see that the even dimensional case cannot be quasi-classical. If we symmetrize $C_{2}(2 m)$ and write it in matrix form, we obtain that it is non-degenerate if and only if $a_{0} a_{1} \neq 0$. Now, considering the differential operator $\widehat{X}_{2}$ we obtain

$\widehat{X}_{2}\left(C_{2}(2 m)\right)=-x_{3} \frac{\partial C_{2}(2 m)}{\partial x_{1}}-C_{2 j}^{k} x_{k} \frac{\partial C_{2}(2 m)}{\partial x_{j}}=-2 a_{0} x_{1} x_{3}-C_{2 j}^{k} x_{k} \frac{\partial C_{2}(2 m)}{\partial x_{j}}$

Since $\mathfrak{n}$ is nilpotent, we have $X_{1}, X_{2} \notin[\mathfrak{n}, \mathfrak{n}]$. This means that if $C_{2}(2 m)$ is a solution of $\widehat{X}_{2}$, then the term $-2 a_{0} x_{1} x_{3}$ must cancel, i.e., $a_{0}=0$. But this implies that the quadratic operator is degenerate, thus $\mathfrak{n}$ is not quasi-classical.

\section{Classification of QCLAs up to dimension 6}

The classification of low dimensional quasi-classical Lie algebras follows from the general classification of real Lie algebras and their invariants (Patera et al., 1976; CampoamorStursberg, 2005; Boyko et al., 2006). By the preceding results, it suffices to consider the indecomposable algebras. The quasi-classical solvable Lie algebras in dimension $n \leq 6$ are given in Table 1. It will turn out that in low dimension, the contractions of reductive algebras allow to recover the quasi-classical algebras.

We recall that a contraction $\mathfrak{g} \rightsquigarrow \mathfrak{g}^{\prime}$ of a Lie algebra $\mathfrak{g}$ onto $\mathfrak{g}^{\prime}$ is given by the brackets

$$
[X, Y]^{\prime}:=\lim _{t \rightarrow \infty} \Phi_{t}^{-1}\left[\Phi_{t}(X), \Phi_{t}(Y)\right]
$$

where $\Phi_{t}$ is an automorphism of $\mathfrak{g}$ for all $t<\infty$. The physically most interesting type of contractions are the so called generalized Inönü-Wigner contractions (short gen. IW), introduced in (Weimar-Woods, 2000), and given by automorphims of the type

$$
\Phi_{t}\left(X_{i}\right)=t^{-n_{i}} X_{i}, \quad n_{i} \in \mathbb{Z} .
$$

Now, if $F\left(X_{1}, \ldots, X_{n}\right)=\alpha^{i_{1} \ldots i_{p}} X_{i_{1}} \ldots X_{i_{p}}$ is a Casimir operator of degree $p$, then the transformed invariant takes the form

$$
F\left(\Phi_{t}\left(X_{1}\right), . ., \Phi_{t}\left(X_{n}\right)\right)=t^{n_{i_{1}}+\ldots+n_{i_{p}}} \alpha^{i_{1} \ldots i_{p}} X_{i_{1}} \ldots X_{i_{p}} .
$$

Now, taking

$$
M=\max \left\{n_{i_{1}}+\ldots+n_{i_{p}} \mid \alpha^{i_{1} . i_{p}} \neq 0\right\}
$$


the limit

$F^{\prime}\left(X_{1}, . ., X_{n}\right)=\lim _{t \rightarrow \infty} t^{-M} F\left(\Phi_{t}\left(X_{1}\right), \ldots, \Phi_{t}\left(X_{n}\right)\right)=\sum_{n_{i_{1}}+\ldots+n_{i_{p}}=M} \alpha^{i_{1} \ldots i_{p}} X_{i_{1}} \ldots X_{i_{p}}$

provides a Casimir operator of degree $p$ of the contraction $\mathfrak{g}^{\prime}$. This procedure allows to obtain invariants of contractions from invariants in the contracting Lie algebra (WeimarWoods, 1996).

Proposition 5 Any non-semisimple quasi-classical Lie algebra $\mathfrak{g}$ of dimension $n \leq 6$ is a generalized Inönü contraction of a reductive Lie algebra.

Proof. For the Lie algebras $A_{4,8}, A_{4,10}, A_{5,3}, A_{6,3}$ we obtain the contraction explicitly, while for the six dimensional solvable algebras of Table 1 we proceed by means of deformation theory (Goze, 1988).

The contraction $\mathfrak{s l}(2, \mathbb{R}) \oplus L_{1} \rightsquigarrow A_{4,8}$ is described in (Huddleston, 1978), while $\mathfrak{s o}(3) \oplus L_{1} \rightsquigarrow A_{4,10}$, not contained in that list, is given by the automorphism

$$
\Phi_{t}\left(X_{1}\right)=\frac{1}{t^{2}} X_{1}, \quad \Phi_{t}\left(X_{2}\right)=\frac{1}{t} X_{2}, \quad \Phi_{t}\left(X_{3}\right)=\frac{1}{t} X_{3}, \quad \Phi_{t}\left(X_{4}\right)=X_{1}+X_{4} .
$$

It is trivial to verify that the contraction is gen. IW. We remark that $A_{4,10}$ is also a contraction of $\mathfrak{s l}(2, \mathbb{R}) \oplus L_{1}$. For the nilpotent Lie algebra $A_{5,3}$ we obtain a gen. IW. contraction $\mathfrak{s o}(3) \oplus 2 L_{1} \rightsquigarrow A_{5,3}$ given by the automorphism

$$
\begin{aligned}
& \Phi_{t}\left(X_{1}\right)=\frac{1}{t^{3}} X_{1}, \quad \Phi_{t}\left(X_{2}\right)=\frac{1}{t^{3}} X_{2}, \quad \Phi_{t}\left(X_{3}\right)=\frac{1}{t^{2}} X_{3}, \\
& \Phi_{t}\left(X_{4}\right)=\frac{1}{t}\left(X_{1}+X_{4}\right), \quad \Phi_{t}\left(X_{5}\right)=\frac{1}{t}\left(X_{2}+X_{5}\right) .
\end{aligned}
$$

Finally, $A_{6,3}$ arises as gen. IW. contraction of $\mathfrak{s o}(3) \oplus 3 L_{1}$ by considering the automorphism

$$
\begin{aligned}
& \Phi_{t}\left(X_{1}\right)=\frac{1}{t^{2}} X_{1}, \quad \Phi_{t}\left(X_{2}\right)=\frac{1}{t^{2}} X_{2}, \quad \Phi_{t}\left(X_{3}\right)=\frac{1}{t^{2}} X_{3}, \\
& \Phi_{t}\left(X_{4}\right)=\frac{1}{t}\left(X_{1}+X_{4}\right), \quad \Phi_{t}\left(X_{5}\right)=\frac{1}{t}\left(X_{2}+X_{5}\right), \quad \Phi_{t}\left(X_{6}\right)=\frac{1}{t}\left(X_{3}+X_{6}\right) .
\end{aligned}
$$

We now turn our attention to the solvable non-nilpotent QCLAs in dimension six. We prove the statement for $\mathfrak{g}_{6,82}^{0, \lambda, \lambda_{1}}$, the argument being the same for the remaining algebras. If the algebra is a contraction, then there exists a deformation that reverses it (Goze, 1988; Weimar-Woods, 2000). Thus we analyze the invertible deformations of $\mathfrak{g}_{6,82}^{0, \lambda, \lambda_{1}}$ and see whether they lead to reductive Lie algebras. Computing the second cohomology group of the Lie algebra (see e.g. (Azcárraga and Izquierdo, 1995)), we find the nontrivial cocycle $\varphi \in H^{2}\left(\mathfrak{g}_{6,82}^{0, \lambda, \lambda_{1}}, \mathfrak{g}_{6,82}^{0, \lambda, \lambda_{1}}\right)$ given by

$$
\varphi\left(X_{1}, X_{3}\right)=X_{3}, \quad \varphi\left(X_{1}, X_{5}\right)=-X_{5}, \quad \varphi\left(X_{2}, X_{4}\right)=\lambda_{1}^{-1} X_{6} .
$$

It is straightforward to see that the formal deformation $\mathfrak{g}_{6,82}^{0, \lambda, \lambda_{1}}+\varphi$ given by the bracket $[X, Y]_{\varphi}=[X, Y]+\varphi(X, Y)$ defines a Lie algebra. Moreover, the derived subalgebra has 
dimension six, thus the deformation is a perfect Lie algebra. Computing the Killing tensor of $\mathfrak{g}_{6,82}^{0, \lambda, \lambda_{1}}+\varphi$ we obtain the matrix

$$
\kappa=\left(\begin{array}{cccccc}
2 & 0 & 0 & 0 & 0 & -2 \lambda_{1} \\
0 & 0 & 0 & -2 \frac{\lambda}{\lambda_{1}} & 0 & 0 \\
0 & 0 & 0 & 0 & 2 & 0 \\
0 & -2 \frac{\lambda}{\lambda_{1}} & 0 & 0 & 0 & 0 \\
0 & 0 & 2 & 0 & 0 & 0 \\
-2 \lambda_{1} & 0 & 0 & 0 & 0 & 2\left(\lambda_{1}^{2}+\lambda^{2}\right)
\end{array}\right)
$$

with $\operatorname{det}(\kappa)=64 \lambda^{4} \lambda_{1}^{-2} \neq 0$ since $\lambda_{1} \lambda \neq 0$. This proves that the deformation $\mathfrak{g}_{6,82}^{0, \lambda, \lambda_{1}}+\varphi$ is semisimple. Now, considering the automorphism

$$
\Phi\left(X_{1}\right)=X_{1}^{\prime}=\frac{1}{t^{2}} X_{1}, \Phi\left(X_{i}\right)=X_{i}^{\prime}=\frac{1}{t} X_{i}, \Phi\left(X_{6}\right)=X_{6}^{\prime}=X_{6}
$$

of $\mathfrak{g}_{6,82}^{0, \lambda, \lambda_{1}}+\varphi$ we get the brackets

$$
\begin{array}{lll}
{\left[X_{1}^{\prime}, X_{3}^{\prime}\right]=\frac{1}{t^{2}} X_{3}^{\prime},} & {\left[X_{1}^{\prime}, X_{5}^{\prime}\right]=-\frac{1}{t^{2}} X_{5}^{\prime},} & {\left[X_{2}^{\prime}, X_{4}^{\prime}\right]=X_{1}^{\prime}+\frac{1}{\lambda_{1} t^{2}} X_{6}^{\prime},} \\
{\left[X_{2}^{\prime}, X_{6}^{\prime}\right]=\lambda \Phi\left(X_{2}\right),} & {\left[X_{3}^{\prime}, X_{5}^{\prime}\right]=X_{1}^{\prime},} & {\left[X_{3}^{\prime}, X_{6}^{\prime}\right]=\lambda_{1} X_{3}^{\prime},} \\
{\left[X_{4}^{\prime}, X_{6}^{\prime}\right]=-\lambda X_{4}^{\prime}, \quad\left[X_{5}^{\prime}, X_{6}^{\prime}\right]=-\lambda_{1} X_{5}^{\prime} .} &
\end{array}
$$

It follows at once that for $t \rightarrow \infty$ we obtain the contraction onto $\mathfrak{g}_{6,82}^{0, \lambda, \lambda_{1}}$. For the remaining algebras, a (invertible) deformation leading to a reductive Lie algebra is indicated in Table 2.

In view of this result, it is natural to ask whether any non-semisimple QCLA is obtained by contraction of a reductive Lie algebra. Although no complete classification of Lie algebras in dimension $n \geq 7$ exists, the following example shows that a QCLA is not necessarily the contraction of a reductive Lie algebra. Consider the ten dimensional Lie algebra $\mathfrak{g}=\mathfrak{s l}(2, \mathbb{R}) \vec{\oplus}_{D_{1} \oplus 2 D_{\frac{1}{2}}} \mathfrak{r}$ given by the brackets

$$
\begin{array}{llll}
{\left[X_{1}, X_{2}\right]=2 X_{2}} & {\left[X_{1}, X_{3}\right]=-2 X_{3}} & {\left[X_{2}, X_{3}\right]=X_{1}} & {\left[X_{1}, X_{4}\right]=X_{4}} \\
{\left[X_{1}, X_{5}\right]=-X_{5}} & {\left[X_{1}, X_{6}\right]=X_{6}} & {\left[X_{1}, X_{7}\right]=-X_{7}} & {\left[X_{1}, X_{8}\right]=2 X_{8}} \\
{\left[X_{1}, X_{10}\right]=-2 X_{10}} & {\left[X_{2}, X_{5}\right]=X_{4}} & {\left[X_{2}, X_{7}\right]=X_{6}} & {\left[X_{2}, X_{9}\right]=2 X_{8}} \\
{\left[X_{2}, X_{10}\right]=X_{9}} & {\left[X_{3}, X_{4}\right]=X_{5}} & {\left[X_{3}, X_{6}\right]=X_{7}} & {\left[X_{3}, X_{8}\right]=X_{9}} \\
{\left[X_{3}, X_{9}\right]=2 X_{10}} & {\left[X_{4}, X_{6}\right]=2 X_{8}} & {\left[X_{4}, X_{7}\right]=X_{9}} & {\left[X_{5}, X_{6}\right]=X_{9}} \\
{\left[X_{5}, X_{7}\right]=2 X_{10}} & & &
\end{array}
$$

This algebra admits the (un-symmetrized) quadratic Casimir operator

$$
C=x_{1} x_{9}+2\left(x_{2} x_{10}-x_{3} x_{8}\right)+x_{4} x_{7}-x_{5} x_{6},
$$

which is non-degenerate. Computing the second cohomology group of $\mathfrak{g}$ we obtain that

$$
\operatorname{dim} Z^{2}(\mathfrak{g}, \mathfrak{g})=\operatorname{dim} B^{2}(\mathfrak{g}, \mathfrak{g})=86,
$$

showing that $H^{2}(\mathfrak{g}, \mathfrak{g})=0$ and therefore that $\mathfrak{g}$ is stable. Thus this algebra does not arise as a contraction (Nijenhuis and Richardson, 1966). We remark that this algebra is the lowest dimensional example of a non-reductive rigid quasi-classical Lie algebra with nonzero Levi subalgebra. 


\section{Generalized Inönü-Wigner contractions onto QCLAs}

Since any Lie algebra contracts onto the abelian Lie algebra of its same dimension, and the latter is trivially quasi-classical, we have that a Lie algebra $\mathfrak{g}$ that contracts onto a quasi-classical algebra $\mathfrak{g}^{\prime}$ is not necessarily endowed with a non-degenerate inner product. However, the question turns more interesting if we discard the abelian algebras, i.e., if we require that $\mathfrak{g}^{\prime}$ is not abelian. Even in this form, the question is still too general and can be answered easily in the negative. Any reductive Lie algebra $\mathfrak{s} \oplus n L_{1}$ is always a contraction of a non quasi-classical Lie algebra. It suffices to consider the algebra $\mathfrak{g}=\mathfrak{s} \oplus \frac{n}{2} r_{2}$ if $n$ is even and $\mathfrak{g}=\mathfrak{s} \oplus \mathfrak{h}_{\frac{n-1}{2}}$ if $n$ is odd, where $r_{2}$ is the non-abelian algebra in dimension 2 and $\mathfrak{h}_{\frac{n-1}{2}}$ is the Heisenberg algebra of dimension $n$. Both algebras are easily seen to contract onto $\mathfrak{s} \oplus n L_{1}$, and none of them is quasi-classical since their quadratic Casimir operators are degenerate.

In order to eliminate these trivial cases, we can reformulate the question in the following form:

Problem: If $\mathfrak{g}^{\prime}$ is an indecomposable quasi-classical Lie algebra and $\mathfrak{g}$ a Lie algebra contracting nontrivially onto it, i.e., $\mathfrak{g} \rightsquigarrow \mathfrak{g}^{\prime}$, under which conditions $\mathfrak{g}$ is also quasiclassical?

First of all, a QCLA can be the contraction of an algebra that has no Casimir operators (in the classical sense) at all. To this extent, let $\mathfrak{r}_{6,38}^{\alpha}$ be the solvable Lie algebra given by the brackets

$$
\begin{array}{lll}
{\left[X_{2}, X_{3}\right]=X_{1},} & {\left[X_{1}, X_{6}\right]=2 \alpha X_{1},} & {\left[X_{2}, X_{6}\right]=\alpha X_{2}+X_{3}+X_{4},} \\
{\left[X_{3}, X_{6}\right]=-X_{2}+\alpha X_{3}+X_{5},} & {\left[X_{4}, X_{6}\right]=\alpha X_{4}+X_{5},} & {\left[X_{5}, X_{6}\right]=-X_{4}+\alpha X_{5} .}
\end{array}
$$

This algebra has two invariants, which can be chosen as

$$
I_{1}=\left(x_{4}^{2}+x_{5}^{2}\right)\left(\frac{x_{4}-i x_{5}}{x_{4}+i x_{5}}\right)^{i \alpha}, \quad I_{2}=x_{1} \exp \left(-2 \alpha \arctan \left(x_{4} x_{5}^{-1}\right)\right) .
$$

Now consider the family of automorphims $f_{t}: \mathfrak{r}_{6,38}^{\alpha} \rightarrow \mathfrak{r}_{6,38}^{\alpha}$ defined by

$$
\begin{aligned}
& f_{t}\left(X_{i}\right)=X_{i}^{\prime}=t^{2} X_{i}, \quad i=1,4,5 \\
& f_{t}\left(X_{i}\right)=X_{i}^{\prime}=t X_{i}, \quad i=2,3,6 .
\end{aligned}
$$

The brackets over the transformed basis are:

$$
\begin{array}{lll}
{\left[X_{2}^{\prime}, X_{3}^{\prime}\right]=X_{1}^{\prime},} & {\left[X_{1}^{\prime}, X_{6}^{\prime}\right]=2 t \alpha X_{1}^{\prime},} & {\left[X_{2}^{\prime}, X_{6}^{\prime}\right]=\alpha t X_{2}^{\prime}+t X_{3}^{\prime}+X_{4}^{\prime},} \\
{\left[X_{3}^{\prime}, X_{6}^{\prime}\right]=-t X_{2}^{\prime}+\alpha t X_{3}^{\prime}+X_{5}^{\prime},} & {\left[X_{4}^{\prime}, X_{6}^{\prime}\right]=\alpha t^{2} X_{4}^{\prime}+t X_{5}^{\prime},} & {\left[X_{5}^{\prime}, X_{6}^{\prime}\right]=-t X_{4}^{\prime}+\alpha t X_{5}^{\prime} .}
\end{array}
$$

For $t \rightarrow 0$, all brackets but

$$
\left[X_{2}^{\prime}, X_{3}^{\prime}\right]=X_{1}^{\prime}, \quad\left[X_{2}^{\prime}, X_{6}^{\prime}\right]=X_{4}^{\prime}, \quad\left[X_{3}^{\prime}, X_{6}^{\prime}\right]=X_{5}^{\prime}
$$

vanish, and the resulting algebra is nilpotent and isomorphic to $A_{6,3}$. The main observation is that the quadratic Casimir operator of $A_{6,3}$ does not arise as the limit of a $\mathfrak{r}_{6,38}^{\alpha}$ invariant. This happens because the contraction does not preserve the number $\mathcal{N}$ of invariants (Campoamor-Stursberg, 2003a). This fact suggests a refinement of the problem: 
Refinement: If $\mathfrak{g}^{\prime}$ is an indecomposable quasi-classical Lie algebra and $\mathfrak{g}$ a Lie algebra contracting nontrivially onto it, such that the number of independent invariants is preserved, i.e., $\mathcal{N}(\mathfrak{g})=\mathcal{N}\left(\mathfrak{g}^{\prime}\right)$, under which conditions $\mathfrak{g}$ is also quasi-classical?

By this assumption, we guarantee that a fundamental system of invariants of the contraction can be obtained by a limiting process of a system of invariants of the contracted algebra (Campoamor-Stursberg, 2004). However, even in this case, a QCLA is not necessarily the contraction of another quasi-classical algebra, as the following example shows: Let $\mathfrak{r}_{6,94}^{-2}$ be the solvable Lie algebra given by

$$
\begin{array}{lll}
{\left[X_{3}, X_{4}\right]=X_{1},} & {\left[X_{2}, X_{5}\right]=X_{1}, \quad\left[X_{3}, X_{5}\right]=X_{2}, \quad\left[X_{2}, X_{6}\right]=-X_{2},} \\
{\left[X_{3}, X_{6}\right]=-2 X_{3}, \quad\left[X_{4}, X_{6}\right]=2 X_{4}, \quad\left[X_{5}, X_{6}\right]=X_{5} .}
\end{array}
$$

It has the cubic Casimir operator $C_{3}=x_{1}^{2} x_{6}+x_{1} x_{2} x_{5}+2 x_{1} x_{3} x_{4}-x_{2}^{2} x_{4}$. Taking the contraction determined by the automorphism

$$
\Phi\left(X_{1}\right)=\frac{1}{t^{2}} X_{1}, \quad \Phi\left(X_{i}\right)=\frac{1}{t} X_{i}, i=2 . .5, \quad \Phi\left(X_{6}\right)=X_{6},
$$

we obtain that

$$
\lim _{t \rightarrow \infty} \frac{1}{t^{4}}\left(C_{3} \circ \Phi\right)=x_{1}\left(x_{1} x_{6}+x_{2} x_{5}+2 x_{3} x_{4}\right),
$$

showing that the contraction is quasi-classical. This situation arises whenever we have a nontrivial centre and a cubic operator that decomposes as the product of a nondegenerate quadratic polynomial with the generator of the centre and some additional cubic term independent of the centre generator that vanishes during the contraction. A similar situation holds for higher dimensional operators and nonzero centre. The remaining case is to see whether a quadratic invariant which involves all generators of the algebra but is degenerate as bilinear form can contract onto a non-degenerate quadratic Casimir operator.

Theorem 1 Let $\mathfrak{g}^{\prime}$ be an indecomposable QCLA and $\mathfrak{g} \rightsquigarrow \mathfrak{g}^{\prime}$ a nontrivial contraction such that

(i) $\mathcal{N}(\mathfrak{g})=\mathcal{N}\left(\mathfrak{g}^{\prime}\right)$

(ii) the non-degenerate quadratic Casimir operator $\widehat{C}$ of $\mathfrak{g}^{\prime}$ is the limit of a quadratic operator $C$ of $\mathfrak{g}$.

Then $C$ is non-degenerate and $\mathfrak{g}$ quasi-classical.

Proof. By assumption, the quadratic Casimir operator $\widehat{C}$ of $\mathfrak{g}^{\prime}$ is obtained by a limiting process from a quadratic Casimir operator of $\mathfrak{g}$. Let $C=g^{i j} X_{i} X_{j}$ be the (symmetrized) quadratic Casimir of $\mathfrak{g}$. Suppose that the automorphim $\Phi_{t}$ of $\mathfrak{g}$ defining the contraction is given by the matrix:

$$
\left(X_{1}^{\prime}, . ., X_{n}^{\prime}\right)=\left(\begin{array}{ccc}
\alpha_{1}^{1} t^{m_{1}^{1}} & \ldots & \alpha_{n}^{1} t^{m_{n}^{1}} \\
\vdots & & \vdots \\
\alpha_{1}^{n} t^{m_{1}^{n}} & \ldots & \alpha_{n}^{n} t^{m_{n}^{n}}
\end{array}\right)\left(\begin{array}{c}
X_{1} \\
\vdots \\
X_{n}
\end{array}\right)
$$


where $m_{i}^{j} \in \mathbb{Z}$ for all $1 \leq i, j \leq n$. Since the matrix is invertible, we can find $\beta_{i}^{k} \in \mathbb{R}$ such that

$$
X_{i}=\beta_{i}^{k} t^{m_{i}^{k}} X_{k}^{\prime}, 1 \leq i \leq n .
$$

Over the transformed basis $\left\{X_{1}^{\prime}, \ldots, X_{n}^{\prime}\right\}$, the operator $C$ takes the form

$$
C=g^{i j} X_{i} X_{j}=g^{i j} \beta_{k}^{i} \beta_{l}^{j} t^{m_{k}^{i}+m_{l}^{j}} X_{i}^{\prime} X_{j}^{\prime} .
$$

Let $M=\max \left\{m_{i}^{j}+m_{k}^{l} \mid 1 \leq i, j, k, l \leq n\right\}$. Let us write $g^{i j}(t):=g^{i j} \beta_{k}^{i} \beta_{l}^{j} t^{m_{k}^{i}+m_{l}^{j}}$ for all $1 \leq i, j \leq n$. These are polynomials in $t$ of degree at most $M$, so that we can find $\gamma_{p}^{i j} \in \mathbb{R}$ for $p=0, . ., M$ such that $g^{i j}(t)=\gamma_{0}^{i j} t^{M}+\gamma_{1}^{i j} t^{M-1}+\ldots+\gamma_{M}^{i j}$. We can therefore rewrite (16) in matrix form $C=\left(X_{1}^{\prime}, . ., X_{n}^{\prime}\right) A\left(X_{1}^{\prime}, . ., X_{n}^{\prime}\right)^{T}$, where $A$ is the (polynomial) matrix

$$
A=\left(\begin{array}{ccc}
\gamma_{0}^{11} t^{M}+\gamma_{1}^{11} t^{M-1}+\ldots+\gamma_{M}^{11} & \ldots & \gamma_{0}^{n 1} t^{M}+\gamma_{1}^{n 1} t^{M-1}+\ldots+\gamma_{M}^{n 1} \\
\vdots & & \vdots \\
\gamma_{0}^{1 n} t^{M}+\gamma_{1}^{1 n} t^{M-1}+\ldots+\gamma_{M}^{1 n} & \ldots & \gamma_{0}^{n n} t^{M}+\gamma_{1}^{n n} t^{M-1}+\ldots+\gamma_{M}^{n n}
\end{array}\right)
$$

Using elementary properties of determinants, $\operatorname{det}(A)$ can be written as a polynomial in $t$ of degree at most $2 M$ :

$$
\operatorname{det}(A)=\Delta_{0} t^{2 M}+\Delta_{1} t^{2 M-1}+\ldots+\Delta_{2 M-1} t+\Delta_{2 M} .
$$

Now, if $C$ were a degenerate operator, then for all $t$ the rank of $A$ is less than $n$, and in particular $\operatorname{det}(A)=0$. But since (18) has at most $2 M$ roots, degeneracy implies that $\Delta_{k}=0$ for $k=0, . ., 2 M$. By contraction, we have that

$$
\lim _{t \rightarrow \infty} \frac{1}{t^{M}} C=\widehat{C}
$$

is the quadratic invariant of $\mathfrak{g}^{\prime}$. However, if all $\Delta_{k}$ vanish, then $\widehat{C}$ must also be a degenerate operator, contradicting the assumption. Therefore, non-degeneracy of $\widehat{C}$ is only possible if $C$ is non-degenerate, proving that $\mathfrak{g}$ is also a quasi-classical Lie algebra.

Corollary 2 Let $\mathfrak{g} \rightsquigarrow \mathfrak{g}^{\prime}$ be a non-trivial generalized Inönü-Wigner contraction and $C$ a non-degenerate quadratic Casimir operator of $\mathfrak{g}$. If $C$ remains invariant by the contraction, then $\mathfrak{g}^{\prime}$ is quasi-classical.

\section{Contraction of Yang-Mills equations}

In view of the preceding theorem, it is worthy to analyze what happens if one tries to compare the behavior of the Yang-Mills equations over quasi-classical Lie algebras $\mathfrak{g}$ and $\mathfrak{g}^{\prime}$ related by a non-trivial contraction $\mathfrak{g} \rightsquigarrow \mathfrak{g}^{\prime}$. Let $C=g^{i j} X_{i} X_{j}$ be the quadratic nondegenerate Casimir operator of $\mathfrak{g}$. If we consider a generalized Inönü-Wigner contraction

$$
X_{i}^{\prime}=t^{-n_{i}} X_{i}, 1 \leq i \leq n,
$$

then over the transformed basis the operator has the form

$$
C^{\prime}=g^{i j} t^{n_{i}+n_{j}} X_{i}^{\prime} X_{j}^{\prime} .
$$


Let $M=\max \left\{n_{i}+n_{j} \mid g^{i j} \neq 0\right\}$. According to this, the Casimir operator $C^{\prime}$ can be decomposed as

$$
C^{\prime}=\sum_{n_{i}+n_{j}=M} t^{n_{i}+n_{j}} g^{i j} X_{i}^{\prime} X_{j}^{\prime}+\sum_{n_{i}+n_{j}<M} t^{n_{i}+n_{j}} g^{i j} X_{i}^{\prime} X_{j}^{\prime} .
$$

Since $t^{-M} C^{\prime}$ is also a non-degenerate quadratic operator on $\mathfrak{g}$, it follows from Theorem 1 that

$$
\lim _{t \rightarrow \infty} \frac{1}{t^{M}} C^{\prime}=\sum_{n_{i}+n_{j}=M} g^{i j} X_{i}^{\prime} X_{j}^{\prime}
$$

is a non-degenerate quadratic Casimir operator of $\mathfrak{g}^{\prime}$. In particular, the non-degenerate bilinear symmetric associative form on $\mathfrak{g}^{\prime}$ is given by the matrix $\left(g^{i j}\right)$, where the condition $n_{i}+n_{j}=M$ holds. Over the transformed basis $\left\{X_{1}^{\prime}, . ., X_{n}^{\prime}\right\}$ of $\mathfrak{g}$ we have the gauge fields

$$
\begin{aligned}
& A_{\mu}(x)=t^{n_{\alpha}} X_{\alpha} A_{\mu}^{\alpha}(x), \\
& F_{\mu \nu}(x)=\partial_{\mu} A_{v}(x)-\partial_{\nu} A_{\mu}(x)+\left[A_{\mu}(x), A_{\nu}(x)\right],
\end{aligned}
$$

where in this case

$$
\left[A_{\mu}(x), A_{\nu}(x)\right]=t^{n_{r}-n_{p}-n_{q}} C_{p q}^{r} A_{\mu}^{p}(x) A_{\nu}^{q}(x) X_{r}^{\prime} .
$$

Taking into account that the latter bracket can be decomposed as

$$
\left[A_{\mu}(x), A_{\nu}(x)\right]=\sum_{n_{r}-n_{p}-n_{q}=0} C_{p q}^{r} A_{\mu}^{p}(x) A_{\nu}^{q}(x) X_{r}^{\prime}+\sum_{n_{r}-n_{p}-n_{q}<0} t^{n_{r}-n_{p}-n_{q}} C_{p q}^{r} A_{\mu}^{p}(x) A_{\nu}^{q}(x) X_{r}^{\prime},
$$

we obtain that for $t \rightarrow \infty$ the limit of $F_{\mu \nu}(x)$ equals

$$
F_{\mu \nu}(x)^{\prime} \lim _{t \rightarrow \infty} F_{\mu \nu}(x)=\partial_{\mu} A_{v}(x)-\partial_{\nu} A_{\mu}(x)+\sum_{n_{r}-n_{p}-n_{q}=0} C_{p q}^{r} A_{\mu}^{p}(x) A_{\nu}^{q}(x) X_{r}^{\prime} .
$$

On the other hand, the Lagrangian on $\mathfrak{g}$ is given by

$$
L(x)=\sum_{n_{i}+n_{j}=M} g^{i j} F_{\mu \nu, i} F_{, j}^{\mu \nu}+\sum_{n_{i}+n_{j}<M} t^{n_{i}+n_{j}-M} g^{i j} F_{\mu \nu, i} F_{, j}^{\mu \nu} .
$$

Again, considering the limit, $L(x)$ goes over to

$$
L^{\prime}(x)=\lim _{t \rightarrow \infty} L(x)=\sum_{n_{i}+n_{j}=M} g^{i j} F_{\mu \nu, i} F_{, j}^{\mu \nu},
$$

equation that reproduces the Lagrangian of $\mathfrak{g}^{\prime}$ with respect to the bilinear form defined by the quadratic operator $\lim _{t \rightarrow \infty} \frac{1}{t^{M}} C^{\prime}$. In this sense, the equations of motion of the Yang-Mills equations of $\mathfrak{g}^{\prime}$ can be recovered from the limit (for $t \rightarrow \infty$ ) of the equations of motion (4) corresponding to $\mathfrak{g}$. It is interesting that by this contraction procedure, we can obtain a large hierarchy of Lagrangians corresponding to non-isomorphic Lie algebras, starting from a suitable Lie algebra.

To illustrate this fact, consider the contraction $\mathfrak{s o}(3,1) \rightsquigarrow \mathfrak{g}_{6,93}^{\alpha=0, \eta \geq 2}$ of the Lorentz algebra onto the quasi-classical solvable Lie algebra $\mathfrak{g}_{6,93}^{0, \eta}$ (see Table 2 ). We choose a basis $\left\{X_{1}, . ., X_{6}\right\}$ of the Lorentz algebra such that the brackets are given by

$$
\begin{aligned}
& {\left[X_{1}, X_{2}\right]=\eta^{2} X_{4}, \quad\left[X_{1}, X_{3}\right]=-\eta^{2} X_{5}, \quad\left[X_{1}, X_{4}\right]=\eta^{2} X_{2}, \quad\left[X_{1}, X_{5}\right]=-\eta^{2} X_{3},} \\
& {\left[X_{2}, X_{4}\right]=X_{1}+X_{6}, \quad\left[X_{2}, X_{5}\right]=-\eta X_{6}, \quad\left[X_{2}, X_{6}\right]=X_{4}+\eta X_{5}, \quad\left[X_{3}, X_{4}\right]=\eta X_{6},} \\
& {\left[X_{3}, X_{5}\right]=X_{1}, \quad\left[X_{3}, X_{6}\right]=\eta X_{4}, \quad\left[X_{4}, X_{6}\right]=X_{2}-\eta X_{3}, \quad\left[X_{5}, X_{6}\right]=-\eta X_{2},}
\end{aligned}
$$


where $\eta \geq 2$. Considering the automorphism given by

$$
X_{1}^{\prime}=\frac{1}{t^{2}} X_{1}, \quad X_{i}^{\prime}=\frac{1}{t} X_{i},(2 \leq i \leq 5), \quad X_{6}^{\prime}=X_{6}
$$

it follows at once that for $t \rightarrow \infty$ we obtain the quasi-classical solvable Lie algebra $\mathfrak{g}_{6,93}^{0, \eta}$ of Table 1. Over the transformed basis $\left\{X_{1}^{\prime}, . ., X_{6}^{\prime}\right\}$ the (symmetrized) quadratic Casimir operator of $\mathfrak{s o}(3,1)$ can be chosen as

$$
C=-2 X_{1}^{\prime} X_{6}^{\prime}+2 \eta\left(X_{2}^{\prime} X_{3}^{\prime}+X_{4}^{\prime} X_{5}^{\prime}\right)+\left(X_{4}^{\prime 2}-X_{2}^{\prime 2}\right)-\frac{1}{t^{2}} X_{6}^{\prime 2},
$$

which in the limit provides the quadratic invariant of $\mathfrak{g}_{6,93}^{0, \eta}$. Constructing the Lagrangian from the bilinear form $g^{a b}$ determined by the previous Casimir operator, we obtain

$L(x)=-F_{\mu \nu, 1}(x) F_{6}^{\mu \nu}+2 \eta\left(F_{\mu \nu, 2}(x) F_{3}^{\mu \nu}+F_{\mu \nu, 4} F_{, 5}^{\mu \nu}\right)+\left(F_{\mu \nu, 4} F_{, 4}^{\mu \nu}-F_{\mu \nu, 2} F_{, 2}^{\mu \nu}\right)-\frac{1}{t^{2}} F_{\mu \nu, 6}(x) F_{6}^{\mu \nu}$,

where in this case

$$
\begin{aligned}
& F_{\mu \nu}(x)=\partial_{\mu} A_{\nu}(x)-\partial_{\nu} A_{\mu}(x)+\left(A_{\mu}^{2}(x) A_{\nu}^{4}(x)+A_{\mu}^{3}(x) A_{\nu}^{5}(x)\right) X_{1}^{\prime}+ \\
& \left(\frac{\eta^{2}}{t} A_{\mu}^{1}(x) A_{\nu}^{4}(x)+A_{\mu}^{4}(x) A_{\nu}^{6}(x)-\eta A_{\mu}^{5}(x) A_{\nu}^{6}(x)\right) X_{2}^{\prime}-\left(\frac{\eta^{2}}{t} A_{\mu}^{1}(x) A_{\nu}^{5}(x)+\eta A_{\mu}^{4}(x) A_{\nu}^{6}(x)\right) X_{3}^{\prime}+ \\
& \left(\frac{\eta^{2}}{t} A_{\mu}^{1}(x) A_{\nu}^{4}(x)+A_{\mu}^{2}(x) A_{\nu}^{6}(x)+\eta A_{\mu}^{3}(x) A_{\nu}^{6}(x)\right) X_{4}^{\prime}+\left(\frac{-\eta^{2}}{t} A_{\mu}^{1}(x) A_{\nu}^{3}(x)+\eta A_{\mu}^{2}(x) A_{\nu}^{6}(x)\right) X_{5}^{\prime}+ \\
& \left(-\frac{\eta}{t^{2}} A_{\mu}^{2}(x) A_{\nu}^{5}(x)+\frac{\eta}{t^{2}} A_{\mu}^{3}(x) A_{\nu}^{4}(x)+\frac{1}{t^{2}} A_{\mu}^{2}(x) A_{\nu}^{4}(x)\right) X_{6}^{\prime}
\end{aligned}
$$

In the limit, $t \rightarrow \infty$ seven terms in (31) vanish, and the result is the corresponding $F_{\mu \nu}(x)$ for the contraction $\mathfrak{g}_{6,93}^{0, \eta}$. We remark that, although (301) and (31) are related to the Lorentz algebra for all values of $\eta$, after the contraction the Lagrangian and the gauge fields correspond to non-isomorphic Lie algebras. Therefore the contraction of the Yang-Mills equations of a simple algebra give rise to the corresponding problem on a parameterized family of solvable Lie algebras. In this construction, the parameter $\eta$ introduced as a scaling factor before contraction, becomes an essential parameter after it, determining the isomorphism class of the Lie algebra on which the gauge fields take their values. It should be expected that this parameter plays also a role in comparing the solutions for the different non-isomorphic contractions, starting from the solutions to the original equation.

\section{Concluding remarks}

After analyzing various properties of general quasi-classical Lie algebras, concretely nilpotent and non-solvable Lie algebras with nontrivial Levi subalgebra, and classifying them in low dimension (up to dimension 6 for the solvable case, and 9 for the non-solvable case), we have shown that a quasi-classical algebra is not necessarily the contraction of a reductive algebra, as suggested by the classification in low dimension. The existence of stable QCLAs that are not reductive leads to search for criteria to ensure that a quasi-classical algebra is the contraction of another Lie algebra with the same property. 
Discarding the trivial abelian case, we have seen that non-degenerate quadratic Casimir operators may arise in different forms, from algebras having only pure transcendental invariants or having Casimir invariants of third or higher order. The existence of a non-trivial centre plays a central role, since it allows higher order operators to split into the product of a non-degenerate quadratic invariant and a linear one. We remark that this decomposability pattern is typical in the contraction of Casimir operators of Lie algebras (Campoamor-Stursberg, 2006). However, in the case that the (non-degenerate) quadratic Casimir invariant of the contraction is obtained as the limit of a quadratic operator, this necessarily implies that the contracting algebra also possesses a nondegenerate form. This fact is applied to compare the corresponding Yang-Mills equations before and after contraction, and provides parameterized families of Lagrangians related to non-isomorphic Lie algebras in the contraction. This fact allows, under suitable conditions, to analyze the solutions for these families as a limit of the solutions before applying the limit process. In the case of contractions of reductive Lie algebras, the terms that vanish during the contraction are responsible for the appearance of ghost states when quantized. This method of generating families from one fixed algebra could be of interest for the problem of existence of flat potentials or the asymptotics approach applied to contractions of simple compact Lie algebras (Bohr and Buchner, 1986). In particular, for special values of the parameters some additional features could appear, such as non-flatness of Yang-Mills potentials (Mundt, 1993).

\section{REFERENCES}

Azcárraga, J. A. de, and Izquierdo, J. M. (1995). Lie Groups, Lie Algebras, Cohomology and some Aplications to Physics, (Cambridge: Cambridge University Press).

Bollini, C. G., and Giambigi, J. J. (1984). Zeitschrift der Physik, C22, 257.

Bohr, H., and Buchner, K. (1986). Tensor N. S., 43, 66.

Boyko, V., Patera, J., and Popovych, R. (2006). Journal of Physics A: Mathematical and General, 39, 5749.

Campoamor-Stursberg, R. (2003a). Acta Physica Polonica, B34, 3901.

Campoamor-Stursberg, R. (2003b). Journal of Physics A: Mathematical and General, 36, 1357.

Campoamor-Stursberg, R. (2004). Physics Letters, A327, 138.

Campoamor-Stursberg, R. (2005). Algebra Colloquium, 12, 497.

Campoamor-Stursberg, R. (2006). In Oberwolfach Reports, 3, 174.

Casimir, H. (1931). Verhandelingen der Koninklijke Akademie van Wetenschappen te Amsterdam, 34, 144.

Das, A. (1989). Integrable models, (Singapur: World Scientific).

Favre, G., and Santharoubane, L. J. (1987). Journal of Algebra, 105, 451. 
Goze, M. (1988). In Deformation Theory of Algebras and Structures and Applications, (Amsterdam: Kluwer), p265.

Huddleston, P. L. (1978). Journal of Mathematical Physics, 19, 1645.

Mundt, E. (1993). Seminar Sophus Lie, 3, 107.

Myung, H. C. (1986). Mal'cev admissible algebras, (Boston: Birkhäuser).

Nijenhuis, A., and Richardson, R. W. (1966). Bulletin of the American Mathematical Society, 72, 1.

Okubo, S. (1979). Hadronic Journal, 3, 1.

Okubo, S. (1998). Journal of Physics A: Mathematical and General, 31, 7603.

Okubo, S., and Kamiya, N. (2002). Communications in Algebra, 30, 3825.

Patera, J., Sharp, R. T., Winternitz, P., and Zassenhaus, H. (1976). Journal of Mathematical Physics, 17, 986.

Popov, A. D. (1991). Teoreticheskaya i Matematicheskaya Fizika, 43, 402.

Schimming, R., and Mundt, E. (1992). Journal of Mathematical Physics, 33, 4250.

Slavnov, A. A., and Faddeev, L. D. (1978). Vvedenie v Kvantovuyu Teoriyu Kalibrovochnykh Polei', (Moskva: Nauka).

Turkowski, P. (1988). Journal of Mathematical Physics, 29, 2139.

Turkowski, P. (1992). Linear Algebra and Applications, 171, 197.

Trofimov, V. V. (1983). Trudy Semimara po Vektornomu i Tenzornomu Analizu, 12,84 .

Weimar-Woods, E. (1996). In Proc. XXI Int. Colloq. Group Theoretical Methods in Physics (Goslar), vol 1, (Singapore: World Scientific), p 132.

Weimar-Woods, E. (2000). Reviews in Mathematical Physics, 12, 1505. 
Table 1. Indecomposable solvable QCLAs in dimension $\leq 6$

\begin{tabular}{|c|c|c|c|}
\hline $\mathfrak{g}$ & Brackets & & $\begin{array}{l}\text { Quadratic Casimir } \\
\text { (non-symmetrized) }\end{array}$ \\
\hline$\overline{A_{4,8}}$ & $\begin{array}{l}{\left[X_{2}, X_{3}\right]=X_{1}} \\
{\left[X_{3}, X_{4}\right]=-X_{3}}\end{array}$ & {$\left[X_{2}, X_{4}\right]=X_{2}$} & $x_{2} x_{3}-x_{1} x_{4}$ \\
\hline$A_{4,10}$ & $\begin{array}{l}{\left[X_{2}, X_{3}\right]=X_{1}} \\
{\left[X_{3}, X_{4}\right]=X_{2}}\end{array}$ & {$\left[X_{2}, X_{4}\right]=-X_{3}$} & $x_{2}^{2}+x_{3}^{2}+2 x_{1} x_{4}$ \\
\hline$A_{5,3}$ & $\begin{array}{l}{\left[X_{3}, X_{4}\right]=X_{2}} \\
{\left[X_{4}, X_{5}\right]=X_{3}}\end{array}$ & {$\left[X_{3}, X_{5}\right]=X_{1}$} & $x_{3}^{2}+2 x_{2} x_{5}-2 x_{1} x_{4}$ \\
\hline$A_{6,3}$ & $\begin{array}{l}{\left[X_{1}, X_{2}\right]=X_{6}} \\
{\left[X_{2}, X_{3}\right]=-X_{5}}\end{array}$ & {$\left[X_{1}, X_{3}\right]=X_{4}$} & $x_{1} x_{5}-x_{2} x_{4}+x_{3} x_{6}$ \\
\hline $\begin{array}{l}\mathfrak{g}_{6,82}^{\alpha=0} \\
\lambda \lambda_{1} \neq 0\end{array}$ & $\begin{array}{l}{\left[X_{2}, X_{4}\right]=X_{1}} \\
{\left[X_{2}, X_{6}\right]=\lambda X_{2}} \\
{\left[X_{4}, X_{6}\right]=-\lambda X_{4}}\end{array}$ & $\begin{array}{l}{\left[X_{3}, X_{5}\right]=X_{1}} \\
{\left[X_{3}, X_{6}\right]=\lambda_{1} X_{3}} \\
{\left[X_{5}, X_{6}\right]=-\lambda_{1} X_{5}}\end{array}$ & $\lambda x_{2} x_{4}+\lambda_{1} x_{3} x_{5}-x_{1} x_{6}$ \\
\hline $\begin{array}{l}\mathfrak{g}_{6,83}^{\alpha=0} \\
\lambda \neq 0\end{array}$ & $\begin{array}{l}{\left[X_{2}, X_{4}\right]=X_{1}} \\
{\left[X_{2}, X_{6}\right]=\lambda X_{2}+X_{3}} \\
{\left[X_{3}, X_{6}\right]=\lambda X_{3}} \\
{\left[X_{5}, X_{6}\right]=-X_{4}-\lambda X_{5}}\end{array}$ & $\begin{array}{l}{\left[X_{3}, X_{5}\right]=X_{1}} \\
{\left[X_{4}, X_{6}\right]=-\lambda X_{4}}\end{array}$ & $\lambda\left(x_{2} x_{4}+x_{3} x_{5}\right)+x_{3} x_{4}-x_{1} x_{6}$ \\
\hline $\begin{array}{l}\mathfrak{g}_{6,88}^{\alpha=0} \\
\nu_{0} \neq 0\end{array}$ & $\begin{array}{l}{\left[X_{2}, X_{4}\right]=X_{1}} \\
{\left[X_{2}, X_{6}\right]=\mu_{0} X_{2}+v_{0} X_{3}}\end{array}$ & $\begin{array}{l}{\left[X_{3}, X_{5}\right]=X_{1}} \\
{\left[X_{3}, X_{6}\right]=\mu_{0} X_{3}-v_{0} X_{2}}\end{array}$ & $\mu_{0}\left(x_{2} x_{4}+x_{3} x_{5}\right)-x_{1} x_{6}+$ \\
\hline$\mu_{0} \neq 0$ & {$\left[X_{4}, X_{6}\right]=v_{0} X_{5}-\mu_{0} X_{4}$} & {$\left[X_{5}, X_{6}\right]=-v_{0} X_{4}-\mu_{0} X_{5}$} & $+v_{0}\left(x_{3} x_{4}-x_{2} x_{5}\right)$ \\
\hline $\begin{array}{l}\mathfrak{g}_{6,89}^{\alpha=0} \\
s \nu_{0} \neq 0\end{array}$ & $\begin{array}{l}{\left[X_{2}, X_{4}\right]=X_{1}} \\
{\left[X_{2}, X_{6}\right]=s X_{2}} \\
{\left[X_{4}, X_{6}\right]=-s X_{4}}\end{array}$ & $\begin{array}{l}{\left[X_{3}, X_{5}\right]=X_{1}} \\
{\left[X_{3}, X_{6}\right]=v_{0} X_{5}} \\
{\left[X_{5}, X_{6}\right]=-v_{0} X_{3}}\end{array}$ & $2 x_{1} x_{6}-2 s x_{2} x_{4}-v_{0}\left(x_{3}^{2}+x_{5}^{2}\right)$ \\
\hline $\begin{array}{l}\mathfrak{g}_{6,90}^{\alpha=0} \\
\nu_{0} \neq 0\end{array}$ & $\begin{array}{l}{\left[X_{2}, X_{4}\right]=X_{1}} \\
{\left[X_{2}, X_{6}\right]=X_{4}} \\
{\left[X_{4}, X_{6}\right]=X_{2}}\end{array}$ & $\begin{array}{l}{\left[X_{3}, X_{5}\right]=X_{1}} \\
{\left[X_{3}, X_{6}\right]=v_{0} X_{5}} \\
{\left[X_{5}, X_{6}\right]=-v_{0} X_{3}}\end{array}$ & $2 x_{1} x_{6}+x_{2}^{2}-x_{4}^{2}-v_{0}\left(x_{3}^{2}+x_{5}^{2}\right)$ \\
\hline $\mathfrak{g}_{6,91}$ & $\begin{array}{l}{\left[X_{2}, X_{4}\right]=X_{1}} \\
{\left[X_{2}, X_{6}\right]=X_{4}} \\
{\left[X_{4}, X_{6}\right]=X_{2}}\end{array}$ & $\begin{array}{l}{\left[X_{3}, X_{5}\right]=X_{1}} \\
{\left[X_{3}, X_{6}\right]=X_{5}} \\
{\left[X_{5}, X_{6}\right]=-X_{3}}\end{array}$ & $2 x_{1} x_{6}+x_{2}^{2}-x_{4}^{2}-\left(x_{3}^{2}+x_{5}^{2}\right)$ \\
\hline $\begin{array}{l}\mathfrak{g}_{6,92}^{\alpha=0} \\
\mu_{0}, \nu_{0} \neq 0\end{array}$ & $\begin{array}{l}{\left[X_{2}, X_{4}\right]=X_{1}} \\
{\left[X_{2}, X_{6}\right]=v_{0} X_{3}} \\
{\left[X_{4}, X_{6}\right]=\mu_{0} X_{5}}\end{array}$ & $\begin{array}{l}{\left[X_{3}, X_{5}\right]=X_{1}} \\
{\left[X_{3}, X_{6}\right]=-\mu_{0} X_{2}} \\
{\left[X_{5}, X_{6}\right]=-v_{0} X_{4}}\end{array}$ & $-x_{1} x_{6}-\mu_{0} x_{2} x_{5}+v_{0} x_{3} x_{4}$ \\
\hline $\begin{array}{l}\mathfrak{g}_{6,92}^{*} \\
p=0\end{array}$ & $\begin{array}{l}{\left[X_{2}, X_{4}\right]=X_{5}} \\
{\left[X_{1}, X_{6}\right]=X_{3}} \\
{\left[X_{3}, X_{6}\right]=-X_{1}}\end{array}$ & $\begin{array}{l}{\left[X_{1}, X_{3}\right]=X_{5}} \\
{\left[X_{2}, X_{6}\right]=X_{4}} \\
{\left[X_{4}, X_{6}\right]=-X_{2}}\end{array}$ & $x_{1}^{2}+x_{2}^{2}+x_{3}^{2}+x_{4}^{2}-2 x_{5} x_{6}$ \\
\hline $\begin{array}{l}\mathfrak{g}_{6,93}^{\alpha=0} \\
\nu_{0} \neq 0\end{array}$ & $\begin{array}{l}{\left[X_{2}, X_{4}\right]=X_{1}} \\
{\left[X_{2}, X_{6}\right]=X_{4}+v_{0} X_{5}} \\
{\left[X_{4}, X_{6}\right]=X_{2}-v_{0} X_{3}}\end{array}$ & $\begin{array}{l}{\left[X_{3}, X_{5}\right]=X_{1}} \\
{\left[X_{3}, X_{6}\right]=v_{0} X_{4}} \\
{\left[X_{5}, X_{6}\right]=-v_{0} X_{2}}\end{array}$ & $v_{0}\left(x_{2} x_{3}+x_{4} x_{5}\right)-x_{1} x_{6}+\frac{x_{4}^{2}-x_{2}^{2}}{2}$ \\
\hline
\end{tabular}


Table 2. Deformation of solvable QCLAs to reductive Lie algebras

\begin{tabular}{|c|c|}
\hline$\frac{y}{y}>>>2$ & ycle or derorma \\
\hline 82 & $\varphi\left(X_{1}, X_{3}\right)=X_{3}, \quad \varphi\left(X_{1}, X_{5}\right)=-X_{5}, \quad \varphi\left(X_{2}, X_{4}\right)=\frac{1}{\lambda_{1}} X_{6}$ \\
\hline 83 & $\varphi\left(X_{1}, X_{2}\right)=-\lambda X_{3}, \quad \varphi\left(X_{1}, X_{5}\right)=\lambda X_{4}, \quad \varphi\left(X_{2}, X_{5}\right)=X_{6}$ \\
\hline $\mathfrak{g}_{6,88}$ & $\begin{array}{ccc}\varphi\left(X_{1}, X_{2}\right)=\gamma X_{2}, & \varphi\left(X_{1}, X_{3}\right)=\gamma X_{3}, & \varphi\left(X_{1}, X_{4}\right)=\gamma X_{4}, \\
\varphi\left(X_{1}, X_{5}\right)=\gamma X_{5}, & \varphi\left(X_{2}, X_{4}\right)=\alpha X_{6}, & \varphi\left(X_{2}, X_{5}\right)=\beta X_{6} \\
\varphi\left(X_{3}, X_{4}\right)=-\beta X_{6}, & \varphi\left(X_{3}, X_{5}\right)=\alpha X_{6}, & \gamma=\alpha \mu+\beta \nu, \beta \mu=\alpha \nu\end{array}$ \\
\hline $\mathfrak{g}_{6,89}$ & $\varphi\left(X_{1}, X_{2}\right)=X_{2}, \quad \varphi\left(X_{1}, X_{4}\right)=-X_{4}, \quad \varphi\left(X_{3}, X_{5}\right)=\frac{1}{s} X_{6}$ \\
\hline $\mathfrak{g}_{6,90}$ & $\begin{array}{ccc}\varphi\left(X_{1}, X_{2}\right)=X_{1}, & \varphi\left(X_{1}, X_{6}\right)=-X_{2}, & \varphi\left(X_{2}, X_{6}\right)=X_{6} \\
\varphi\left(X_{3}, X_{4}\right)=-\nu X_{5}, & \varphi\left(X_{3}, X_{5}\right)=X_{4}, \quad \varphi\left(X_{4}, X_{5}\right)=-\nu X_{3}\end{array}$ \\
\hline $\mathfrak{g}_{6,91}$ & $\begin{array}{ccc}\varphi\left(X_{1}, X_{2}\right)=X_{1}, & \varphi\left(X_{1}, X_{6}\right)=-X_{2}, & \varphi\left(X_{2}, X_{6}\right)=X_{6} \\
\varphi\left(X_{3}, X_{4}\right)=-\nu X_{5}, & \varphi\left(X_{3}, X_{5}\right)=X_{4}, \quad \varphi\left(X_{4}, X_{5}\right)=-\nu X_{3} \\
\end{array}$ \\
\hline $\mathfrak{g}_{6,92}$ & $\begin{array}{ccc}\varphi\left(X_{1}, X_{2}\right)=\mu X_{2}, & \varphi\left(X_{1}, X_{3}\right)=\mu X_{3}, & \varphi\left(X_{1}, X_{4}\right)=-\mu X_{4}, \\
\varphi\left(X_{1}, X_{5}\right)=-\mu X_{5}, & \varphi\left(X_{2}, X_{5}\right)=X_{6}, & \varphi\left(X_{3}, X_{4}\right)=-\frac{\mu}{\nu} X_{6}\end{array}$ \\
\hline $\mathfrak{g}_{6,92}^{*}$ & $\begin{array}{lll}\varphi\left(X_{1}, X_{2}\right)=X_{6}, & \varphi\left(X_{1}, X_{5}\right)=X_{2}, \quad \varphi\left(X_{2}, X_{5}\right)=-X_{1}, \\
\varphi\left(X_{3}, X_{4}\right)=X_{6}, \quad \varphi\left(X_{3}, X_{5}\right)=X_{4}, \quad \varphi\left(X_{4}, X_{5}\right)=-X_{3}\end{array}$ \\
\hline $\mathfrak{g}_{6,93}$ & $\begin{array}{ccc}\varphi\left(X_{1}, X_{2}\right)=\nu^{2} X_{4}, & \varphi\left(X_{1}, X_{3}\right)=-\nu^{2} X_{5}, & \varphi\left(X_{1}, X_{4}\right)=\nu^{2} X_{2}, \\
\varphi\left(X_{1}, X_{5}\right)=-\nu^{2} X_{3}, & \varphi\left(X_{2}, X_{4}\right)=X_{6}, & \varphi\left(X_{2}, X_{5}\right)=-\nu X_{6}, \\
\varphi\left(X_{3}, X_{4}\right)=\nu X_{6} & & \end{array}$ \\
\hline
\end{tabular}

